\title{
A importância dos cuidados de enfermagem aos pacientes portadores de Alzheimer
}

\author{
The importance of nursing care for patients with Alzheimer's \\ La importancia de los cuidados de enfermería a los pacientes con Alzheimer
}

Recebido: 06/02/2022 | Revisado: 12/02/2022 | Aceito: 17/02/2022 | Publicado: 26/02/2022

\author{
Brenda Alves Rolim \\ ORCID: https://orcid.org/0000-0001-7151-3020 \\ Faculdade Santa Maria, Brasil \\ E-mail: brendarolim2504@gmail.com \\ Macerlane de Lira Silva \\ ORCID: https://orcid.org/0000-0002-9231-5477 \\ Faculdade Santa Maria, Brasil \\ E-mail: macerlane@ @otmail.com \\ Thárcio Ruston Oliveira Braga \\ ORCID: https://orcid.org/0000-0001-8037-7040 \\ Faculdade Santa Maria, Brasil \\ E-mail: tharcio_ruston@gmail.com \\ Kelli Costa Souza \\ ORCID: https://orcid.org/0000-0002-1811-7499 \\ Faculdade Santa Maria, Brasil \\ E-mail: kelinha.r00@gmail.com \\ Sulaine Cavalcante Rodrigues \\ ORCID: https://orcid.org/0000-0001-7529-3061 \\ Faculdade Santa Maria, Brasil \\ E-mail:sulainecavalcante@ outlook.com \\ Ankilma do Nasimento Andrade Feitosa \\ ORCID: https://orcid.org/0000-0002-4751-2404 \\ Faculdade Santa Maria, Brasil \\ E-mail: ankilmar@hotmail.com
}

\begin{abstract}
Resumo
Objetivos: Analisar a importância dos cuidados de enfermagens aos portadores de Alzheimer. Metodologia: A pesquisa trata-se de um estudo realizado através de uma revisão integrativa da literatura. Utilizou-se as bases de dados de Biblioteca Virtual em Saúde (BVS), Literatura Latino-americana e Caribe em Ciências da Saúde (Lilacs) e Medical Literature Analysis and Retrievel System Online (Medline) e os descritores em ciências da saúde (DECS) Cuidados de Enfermagem; Idoso e Doença de Alzheimer e texto completo em português. Resultados e Discussões: O enfermeiro é o profissional que atua de forma direta nas atividades educacionais prestadas à comunidade, desempenhando uma função de fundamental importância na sociedade com o intuito de prover e promover o empoderamento do cuidado dos usuários, buscando maneiras alternativas que possam ocasionar em atitudes que lhes proporcionem pleno estado de saúde em seu sentido mais amplo. Conclusão: Constata-se que o cuidado de enfermagem vai além do idoso portador da patologia, é necessária uma atenção para aquele que o cuida, pois com toda a responsabilidade sobre o indivíduo, certamente haverá sobrecarga de responsabilidades, o que ocasionará danos à sua saúde. É importante destacar que o cuidador é um ser essencial durante o processo do cuidar.
\end{abstract}

Palavras-chave: Cuidados de enfermagem; Idoso; Doença de Alzheimer.

\begin{abstract}
Objectives: To analyze the importance of nursing care for Alzheimer's patients. Methodology: The research is a study carried out through an integrative literature review. The databases of the Virtual Health Library (VHL), Latin American and Caribbean Literature on Health Sciences (Lilacs) and Medical Literature Analysis and Retrievel System Online (Medline) and the health sciences descriptors (DECS) were used. Nursing care; Elderly and Alzheimer's Disease and full text in Portuguese. Results And Discussion: The nurse is the professional who works directly in the educational activities provided to the community, playing a role of fundamental importance in society in order to provide and promote the empowerment of the elderly. care for users, seeking alternative ways that may lead to attitudes that provide them with full health in the broadest sense. Conclusion: It appears that nursing care goes beyond the elderly with the pathology, attention is needed for the person who takes care of them, because with all the responsibility on the individual, there will certainly be an overload of responsibilities, which will cause damage to their health. . It is important to highlight that the caregiver is an essential being during the care process.
\end{abstract}

Keywords: Nursing care; Elderly; Alzheimer's Disease. 


\begin{abstract}
Resumen
Objetivos: Analizar la importancia de los cuidados de enfermería a los pacientes con Alzheimer. Metodología: La investigación es un estudio realizado a través de una revisión integrativa de la literatura. Se utilizaron las bases de datos de la Biblioteca Virtual en Salud (BVS), Literatura Latinoamericana y del Caribe en Ciencias de la Salud (Lilacs) y Medical Literature Analysis and Retrievel System Online (Medline) y los descriptores de ciencias de la salud (DECS). Ancianos y Enfermedad de Alzheimer y texto completo en portugués Resultados y Discusión: El enfermero es el profesional que actúa directamente en las actividades educativas brindadas a la comunidad, desempeñando un papel de fundamental importancia en la sociedad para proveer y promover el empoderamiento de los ancianos. .cuidar a los usuarios, buscando caminos alternativos que conduzcan a actitudes que les proporcionen salud plena en el sentido más amplio. Conclusión: Parece que el cuidado de enfermería va más allá del anciano con la patología, se necesita atención para la persona que lo cuida, porque con toda la responsabilidad sobre el individuo, seguramente habrá una sobrecarga de responsabilidades, lo que provocará daños a su salud. Es importante resaltar que el cuidador es un ser fundamental durante el proceso de cuidado.
\end{abstract}

Palabras clave: Atención de enfermería; Ancianos; Enfermedad de Alzheimer.

\title{
1. Introdução
}

Com a melhoria das condições de vida e da assistência médica, a expectativa de vida continua aumentando, crescendo também o número de pessoas que sofrem de doenças degenerativas. Entre eles estão alguns pacientes com demência. A demência pode ter diferentes causas e sempre resultará em mudanças graduais de comportamento e dependência das atividades diárias. (Alves et al., 2017).

No entanto, se por um lado temos uma vida mais longa, por outro, somos mais suscetíveis às doenças relacionadas com o envelhecimento. Quando se trata de doenças relacionadas ao envelhecimento, é inevitável falar sobre o Alzheimer.

A Doença de Alzheimer é a principal causa da demência. É uma degeneração primária do sistema nervoso central, ou seja, uma doença que causa atrofia progressiva do cérebro por motivos desconhecidos. A Doença de Alzheimer é caracterizada por uma longa evolução, desde o primeiro erro até a fase grave - entre dez e doze anos em média. Embora o declínio da memória tenha sido observado na fase inicial, com o tempo, as dificuldades vão aumentando, e até as atividades mais simples tornam-se dependentes, como trocar de roupa ou alimentar-se. (Pinto et al., 2017).

Estima-se que $10 \%$ a $15 \%$ dos sintomas em pessoas de 65 anos estejam relacionados à Doença de Alzheimer. A taxa de prevalência saltou a uma taxa de $3 \%$ ao ano até atingir quase $50 \%$ aos 85 anos. Nos estágios iniciais da doença, a perda de memória é mais comum, mas podem ser ignorados sutilmente, porque são confundidos com fenômenos naturais do processo de envelhecimento. Os sinais desta doença incluem o esquecimento de nomes e objetos, os pacientes lembram apenas de coisas que aconteceram no passado, esquecem seu nome e nomes dos seus familiares, tornam-se agressivos e não sabem mais como administrar finanças ou preparar refeições, e têm mudanças frequentes de humor. (Sayeg et al., 2019).

Os profissionais de enfermagem devem se atualizar e acompanhar o desenvolvimento de novas discussões. Com base no conhecimento técnico e científico, a enfermagem pode encontrar uma forma de solucionar alguns diagnósticos de enfermagem, de modo a prescrever orientações importantes para o paciente e para a sua família, por meio de medidas simples de melhoria à saúde para melhorar o prognóstico, a qualidade de vida e evitar complicações. (Sales et al., 2016).

Poucas são as pessoas que estão preparadas em relação ao comprometimento e, consequentemente, a sobrecarga para responsabilizar-se pelo ato de cuidar de uma pessoa acometida por essa doença, tornando-se assim cada vez mais significativo o interesse e a preocupação dos profissionais de enfermagem em possibilitar uma melhor assistência referente aos cuidados com esse indivíduo enfermo. As carências de informações em relação aos cuidados com os portadores da DA acabam influenciando negativamente o desenvolvimento da doença, dessa maneira o conhecimento da enfermagem a respeito do que é a doença, e sobre como proporcionar cuidados específicos, será capaz de beneficiar e favorecer os cuidados prestados ao enfermo, em consequência ocasionará a melhora na qualidade de vida dos idosos e de seus próprios familiares (Silva et al., 2017). 
A função do cuidador é se adaptar ao padrão de vida do portador de DA, tornando-se, assim, muito difícil, pois será necessário privar-se de sua própria individualidade em benefício do próximo, e ainda acaba se deparando com a falta de informações e adequadas orientações referentes aos cuidados (Leite et al., 2016).

No presente momento, muitos acreditam que os indivíduos enfermos ainda possuem o comando sobre seu comportamento, e que, eventualmente, irão voltar ao normal. Portanto, apenas informar sobre a DA não é o suficiente, é necessário disponibilizar uma educação continuada com a finalidade de aperfeiçoar o conhecimento sobre o assunto (Silva et al., 2017).

O enfermeiro é essencial na assistência aos cuidadores com DA, pois visa ao cuidado ao enfermo e à sua própria família, expondo, assim, a qual melhor maneira de superar as alterações funcionais decorrentes da doença e as consequências geradas no ambiente familiar, proporcionando um cuidado integral e com a redução de sofrimentos e desgastes ocasionados pela doença, possibilitando, desta forma, que ambos possam usufruir de uma vida mais equilibrada (Leite et al., 2016).

Sendo que a equipe de enfermagem deve se prontificar em implementar planos elaborados referentes aos cuidados e atividades complementares em conjunto com os cuidadores em prol da melhora da qualidade de vida do idoso, direcionando-os sempre referente sobre a doença e a assistência adequada para cada fase (Poltroniere et al., 2016).

A confirmação da doença origina um grande impacto na vida do indivíduo e no ambiente familiar, porém, através do enfermeiro, poderá proporcionar uma melhor adaptação aos familiares cuidadores na relação com a doença e com o portador de DA. É possível formar grupos de apoio como um espaço de encontro, com a finalidade de troca de experiências e aprendizagem, visando, principalmente, aos familiares de idosos com essa enfermidade, no qual os integrantes possuem a oportunidade de refletir sobre o modo de cuidar através de uma nova perspectiva, encontrando, assim, novas estratégias para a superação das dificuldades e o descobrimento de novas maneiras de como lidar em relação ao cotidiano modificado. Sendo também necessário o planejamento de estratégias para a redução da sobrecarga e da jornada de trabalho, distribuição das responsabilidades e funções (Silva et al., 2017).

No Brasil, existem serviços e programas com o objetivo de prestar atendimento ao idoso acometido pela doença, porém, devido à grande demanda, acaba, assim, ultrapassando a possibilidade de oferta através do poder público, onde ocorre o comprometimento do cuidado ao necessitado. O funcionamento disponível requer a efetuação de gastos econômicos significativos, sendo imprescindível ainda que haja uma criteriosa avaliação prévia do caso antes da indicação do serviço, além da necessidade de uma melhor formação de recursos humanos referentes aos profissionais mais preparados e capacitados (Bulla et al., 2016).

Quando o cuidador familiar é instruído adequadamente, acaba tornando-se qualificado para confrontar com maior segurança os desafios impostos pelo ato de cuidar (Silva et al., 2017).

Esse estudo se justifica em virtude da necessidade em conhecer a importância da assistência de enfermagem na atenção primária ao idoso com Doença de Alzheimer, relatados na literatura.

O objetivo deste estudo é analisar a importância dos cuidados de enfermagens aos portadores de Alzheimer. Mostrar os principais cuidados, diagnósticos e os fatores que afetam a qualidade de vida dos pacientes portadores de Alzheimer.

\section{Metodologia}

Trata-se de um estudo realizado através de uma revisão integrativa da literatura, que é um método que possui o objetivo de identificar, analisar e sintetizar os resultados de pesquisas experimentais e não experimentais, a fim de obter um total entendimento do fenômeno que está sendo analisado. Ela tem sido considerada uma ferramenta única no campo da saúde, já que faz uma síntese dos principais estudos disponíveis sobre uma problemática, promovendo um direcionamento da prática médica, baseado no conhecimento científico (Souza et al., 2010). 
A questão norteadora do trabalho é: Quais os cuidados de enfermagem ao paciente com Doença de Alzheimer?

As buscas foram realizadas com os seguintes descritores: Cuidados de Enfermagem; Idoso e Doença de Alzheimer, com o operador booleano "AND", para ajudar a encontrar os artigos em bancos de dados online. Utilizou-se as bases de dados de Biblioteca Virtual em Saúde (BVS), Literatura Latino-americana e Caribe em Ciências da Saúde (Lilacs) e Medical Literature Analysis and Retrievel System Online (Medline).

Como critérios de inclusão, foram utilizados artigos completos em português, publicados nos últimos 10 anos. Foram utilizados artigos com métodos qualitativos, descritivos, reflexivos e relatos de experiência, que envolviam diretamente a assistência de enfermagem ao idoso com Doença de Alzheimer. Como critério de exclusão, serão excluídos artigos, monografias, resumos, dissertações e artigos que não atendam ao tema da pesquisa. E ainda artigos que não estejam entre o período determinado nos critérios de inclusão.

Por fim, os resultados serão interpretados através de análise crítica e sistemática, apresentados em forma de quadros. Como se tratará de uma revisão bibliográfica com artigos publicados na internet, o presente estudo não executará intervenções diretas em pessoas, podendo ser considerada de risco ínfimo (Sampaio et al., 2007).

Quadro 1 - Seleção dos artigos sobre a importância dos cuidados de enfermagem aos pacientes portadores de Alzheimer.

\begin{tabular}{|l|l|l|}
\hline Descritores Utilizados & $\begin{array}{l}\text { Quantidade de artigos encontrados } \\
\text { por base de dados: }\end{array}$ & $\begin{array}{l}\text { Quantidade de artigos utilizados } \\
\text { após aplicação dos critérios de } \\
\text { inclusão e exclusão: }\end{array}$ \\
\hline $\begin{array}{l}\text { Doença de Alzheimer; Assistência de } \\
\text { enfermagem; Atenção à saúde. }\end{array}$ & $\begin{array}{l}\text { BVS (15) } \\
\text { LILACS ( 56) } \\
\text { PUBMED/MEDLINE ( 7 ) }\end{array}$ & Foram selecionados sete (07) artigos. \\
\hline
\end{tabular}

Fonte: Autores (2022).

\section{Resultados}

De acordo com o exposto no Quadro 2, são apresentadas informações a respeito dos 7 artigos contidos na discussão desta revisão de literatura. Foram interpretados e sintetizados todos os resultados, através de uma comparação dos dados evidenciados na análise dos artigos.

Quadro 2 - Distribuição dos artigos com o autor, ano, título, periódico da publicação e síntese dos resultados.

\begin{tabular}{|c|c|c|c|}
\hline AUTOR/ANO & $\begin{array}{l}\text { TITULO DO } \\
\text { ARTIGO }\end{array}$ & $\begin{array}{c}\text { PERÓDICO } \\
\text { PUBLICAÇÃ̃O }\end{array}$ & SÍNTESE DOS RESULTADOS \\
\hline $\begin{array}{c}\text { ALVES, T. C. T. } \\
\text { F/2017 }\end{array}$ & $\begin{array}{l}\text { Pet do amiloide } \\
\text { cerebral e da } \\
\text { proteína tau no } \\
\text { transtorno } \\
\text { cognitivo leve }\end{array}$ & Psiquiatria Clinica & $\begin{array}{l}\text { Atualmente, o diagnóstico da DA baseia-se na história } \\
\text { clínica do paciente, associada a um exame de RM } \\
\text { estrutural. Essa abordagem é suficiente para avaliação de } \\
\text { casos moderados e graves. Desta forma, a perspectiva de } \\
\text { um método confiável para avaliar a presença de beta- } \\
\text { amilóide cerebral in vivo, de forma não invasiva, em } \\
\text { estágios muito iniciais da DA e eventualmente em estágios } \\
\text { pré-clínicos, mostra-se promissora. }\end{array}$ \\
\hline $\begin{array}{l}\text { APRAHAMIAN, } \\
\text { Ivan; } \\
\text { MARTINELLI, José } \\
\text { Eduardo, } \\
\text { YASSUDA, Mônica } \\
\text { Sanches/2018 }\end{array}$ & $\begin{array}{c}\text { Doença de } \\
\text { Alzheimer: Revisão } \\
\text { da Epidemiologia e } \\
\text { Diagnóstico }\end{array}$ & $\begin{array}{c}\text { Revista da Sociedade } \\
\text { Brasileira de Clínica } \\
\text { Médica }\end{array}$ & $\begin{array}{l}\text { O diagnóstico clínico ainda é o ponto chave para o } \\
\text { diagnóstico dessa forma de demência, excluindo seus } \\
\text { diagnósticos diferenciais. Testes de rastreio cognitivo } \\
\text { como o Mini-Exame do Estado Mental e o Teste do } \\
\text { desenho do relógio são muito importantes para o } \\
\text { diagnóstico de pacientes em estado inicial. }\end{array}$ \\
\hline
\end{tabular}




\begin{tabular}{|c|c|c|c|}
\hline $\begin{array}{l}\text { LEITE, C. D. S. M. } \\
\text { et al./2016 }\end{array}$ & $\begin{array}{l}\text { Conhecimento e } \\
\text { intervenção do } \\
\text { cuidador na Doença } \\
\text { de Alzheimer: uma } \\
\text { revisão da } \\
\text { literatura. }\end{array}$ & $\begin{array}{c}\text { J. bras. psiquiatr., Rio } \\
\text { de Janeiro }\end{array}$ & $\begin{array}{l}\text { Foram eleitos } 12 \text { artigos para análise e leitura na íntegra. } \\
\text { Os artigos foram classificados em três categorias: 1) } \\
\text { Conhecimento acerca do declínio cognitivo e funcional da } \\
\text { Doença de Alzheimer; 2) Conhecimento acerca dos } \\
\text { sintomas psicológicos e comportamentais da demência } \\
\text { (SPCD); 3) Conhecimento acerca do comprometimento da } \\
\text { linguagem. Na maioria dos estudos, verificou-se que a } \\
\text { orientação a respeito do que é a enfermidade e a sua } \\
\text { evolução pode interferir na maneira como o cuidador } \\
\text { planeja e executa as ações de cuidado. }\end{array}$ \\
\hline $\begin{array}{l}\text { POLTRONIERE, S.; } \\
\text { CECCHETTO, F. } \\
\text { H.; SOUZA, } \\
\text { Emiliane Nogueira } \\
\text { de } / 2016\end{array}$ & $\begin{array}{l}\text { Doença de } \\
\text { Alzheimer e } \\
\text { demandas de } \\
\text { cuidados: o que os } \\
\text { enfermeiros sabem? }\end{array}$ & Rev. Gaúcha Enferm & $\begin{array}{l}\text { A saturação dos dados ocorreu com } 11 \text { enfermeiros de } \\
\text { dois hospitais privados. Da análise de conteúdo, } \\
\text { emergiram seis categorias: caracterização da DA, fatores } \\
\text { de risco, formas de diagnóstico, complicações, medicações } \\
\text { para tratamento, cuidados com doente e família. } \\
\text { Observou-se que os enfermeiros possuem um } \\
\text { conhecimento limitado sobre a DA, focando as ações de } \\
\text { cuidado na alteração clínica que motivou a internação } \\
\text { hospitalar. Reconhecem sinais e sintomas, mas se mostram } \\
\text { como figuras coadjuvantes na assistência, quando } \\
\text { deveriam posicionar-se de forma mais autônoma frente ao } \\
\text { cuidado e à atenção à família. }\end{array}$ \\
\hline $\begin{array}{c}\text { SALES, A. C. S. et } \\
a l . / 2016\end{array}$ & $\begin{array}{l}\text { Conhecimento da } \\
\text { equipe de } \\
\text { enfermagem quanto } \\
\text { aos cuidados com } \\
\text { idoso portador da } \\
\text { Doença de } \\
\text { Alzheimer }\end{array}$ & $\begin{array}{c}\text { Revista de } \\
\text { Enfermagem do } \\
\text { Centro Oeste Mineiro }\end{array}$ & $\begin{array}{l}\text { Os resultados demonstraram que há falta de preparo dos } \\
\text { profissionais no cuidado ao idoso e que tal deficiência traz } \\
\text { consequiências para a saúde física e mental dos mesmos. } \\
\text { Foram identificados a partir das falas dos profissionais, } \\
\text { como fatores que interferem no cuidado ao idoso, o } \\
\text { acolhimento durante o processo de admissão; a inclusão } \\
\text { através da realização de eventos culturais e tratamento } \\
\text { com equidade; as exigências físicas e mentais que o } \\
\text { cuidado proporciona à equipe; a falta dos familiares e a } \\
\text { importância do carinho e paciência do cuidador. }\end{array}$ \\
\hline $\begin{array}{l}\text { SANTO, F. H. E.; } \\
\text { SILVA, B.; } \\
\text { CUNHA, S/2018 }\end{array}$ & $\begin{array}{l}\text { Envelhecimento e } \\
\text { morte na concepção } \\
\text { dos idosos e } \\
\text { profissionais de } \\
\text { enfermagem: uma } \\
\text { revisão integrative }\end{array}$ & $\begin{array}{c}\text { REVISTA } \\
\text { TEMÁTICA } \\
\text { KAIRÓS } \\
\text { GERONTOLOGIA }\end{array}$ & $\begin{array}{l}\text { Verificou-se que são variadas as atitudes diante do } \\
\text { envelhecimento e da finitude, dependendo da concepção } \\
\text { de cada pessoa idosa e de cada profissional variável de } \\
\text { acordo com sua história de vida, e de como esse sujeito se } \\
\text { insere na sociedade. }\end{array}$ \\
\hline $\begin{array}{c}\text { SAYEG, } \\
\text { Norton/2019 }\end{array}$ & $\begin{array}{l}\text { Como diagnosticar } \\
\text { e tratar Doença de } \\
\text { Alzheimer }\end{array}$ & R.B.M & $\begin{array}{l}\text { O diagnóstico é difícil, especialmente nos estágios iniciais. } \\
\text { Novos critérios para o diagnóstico têm sido adotados, uma } \\
\text { vez que a doença se inicia alguns anos antes } \\
\text { dos sintomas relacionados às funções cognitivas, } \\
\text { especialmente a perda da memória. }\end{array}$ \\
\hline
\end{tabular}

Fonte: Autores (2022).

\section{Discussão}

Percebe-se, em alguns estudos, o desconhecimento por parte de alguns enfermeiros sobre o processo de alterações ocasionado pela DA, porém, a maioria dos enfermeiros consegue perceber os sinais e sintomas que caracterizam a doença, entretanto, não conhece os aspectos ao manejo da enfermidade. (Camacho et al., 2013).

O cuidado que precisa ser oferecido é mais do que um ato, é uma responsabilização, promovendo um vínculo afetivo. O enfermeiro é o gerente do cuidado, que, ao conhecer o nível das fases e suas consequências, pode planejar e executar diversas atividades para o cuidado com o idoso, paciente e a família. Sendo assim, para o suporte necessário, sugere-se que o enfermeiro conheça o comportamento do idoso com DA, as reações que o cuidador terá diante desses comportamentos e o grau 
de intensidade das fases da doença, e, com isso, crie estratégias de manejo do cuidador para com o paciente, promova ações de enfermagem, com educação em saúde, e em unidade com o cuidador, além de buscar práticas que desenvolvam o diálogo, confiança e, assim, avalie melhor o que se enquadre diante da realidade de cada um. (Soares et al., 2014).

Mattos (2013) destaca alguns dos diagnósticos de enfermagem para a Doença de Alzheimer, como risco para lesão relacionado à falta de atenção aos perigos ambientais; confusão crônica relacionada à incapacidade de avaliar a realidade secundária à degeneração dos neurônios cerebrais; mobilidade física prejudicada, relacionada à instabilidade ao andar; riscos para processos familiares alterados, relacionados aos efeitos da condição sobre os relacionamentos, as responsabilidades e as finanças; manutenção do lar prejudicada, relacionada à incapacidade de cuidar si mesmo e da casa, ou a indisponibilidade da pessoa que presta cuidado; negligência unilateral relacionada à patologia neurológica; déficit no autocuidado relacionado à diminuição da força muscular, desgaste do papel do cuidador relacionado às necessidades múltiplas de cuidados e aos recursos insuficientes. (Oliveira et al., 2014).

As intervenções realizadas pelos profissionais de enfermagem têm o objetivo de preservar ao máximo a capacidade do paciente, e conseguir o melhor desempenho funcional possível em cada estágio da doença, visando sempre ao bem-estar físico e emocional do portador de Alzheimer. (Dias et al., 2014).

$\mathrm{O}$ enfermeiro que se dispõe a estabelecer ações que proporcionem melhoria e qualidade de vida dos portadores da Doença de Alzheimer necessita antes de tudo dispor de paciência, solidariedade e, acima de tudo, respeito ao paciente idoso, estabelecendo um relacionamento afetivo de confiança sobre os cuidados prestados. (Alves et al., 2017).

$\mathrm{O}$ enfermeiro é o profissional que atua de forma direta nas atividades educacionais prestadas à comunidade, desempenhando uma função de fundamental importância na sociedade com o intuito de prover e promover o empoderamento do cuidado dos usuários, buscando maneiras alternativas que possam ocasionar em atitudes que lhes proporcionem pleno estado de saúde em seu sentido mais amplo (Silva et al., 2009).

As atribuições do enfermeiro devem ter foco em diversos aspectos e, para que sejam desempenhadas, o profissional precisa se atualizar e reciclar seus conhecimentos a respeito do cuidado do portador de Doença de Alzheimer (Rodrigues et al., 2007).

O enfermeiro é indispensável no suporte aos cuidadores de idosos com Doenças de Alzheimer, pois visa ao cuidado do indivíduo e sua família, orientando-o para lidar com as adversidades que surgem frente ao cuidado, principais dúvidas a respeito da doença, assim como as principais características evolutivas, e também como lidar com o tratamento, qual a necessidade dos medicamentos administrados, e, ainda, como proceder com os hábitos rotineiros de higiene e alimentação, orientando a melhor forma de superar as modificações funcionais causadas pelas doenças e os impactos gerados no núcleo familiar. (Silva et al., 2018).

Com isso, as diversas leituras realizadas nas diversas pesquisas possibilitaram compreender que ainda se fazem presentes as limitações sobre o conhecimento adquirido pelo profissional da enfermagem sobre a fisiopatologia do Alzheimer. Para isso, é importante que o profissional da enfermagem esteja sempre em formação contínua, adquirindo conhecimento através de especialização profissional, na participação ou promoção de eventos sobre a temática, e no convívio com pacientes e familiares. (Oliveira et al., 2014).

A Enfermagem deve atuar em conjunto com paciente e família, sendo que o enfermeiro é o profissional de saúde que tem um elo entre paciente e família. O cuidado tem como estimular o paciente e envolver a família para o individualismo, o autocuidado, a promoção da segurança física, a redução da ansiedade e agitação. (Soares et al., 2014).

A enfermagem acumulou muito conhecimento e experiência tecnológica desde o início, e hoje desenvolve teorias inter-relacionadas, tentando explicar esses fatos a partir do universo natural. O objetivo da enfermagem é ajudar os seres humanos a atender às suas necessidades humanas básicas. Esta é a entidade de enfermagem. Para que a enfermagem funcione 
de forma eficaz, é necessário desenvolver metodologia de trabalho baseada em métodos científicos. Este método de trabalho da enfermagem é denominado processo de enfermagem (Alves et al., 2017).

As etapas do processo incluem: histórico médico, diagnóstico, plano de cuidados, prescrição de cuidados e evolução. O histórico de enfermagem envolve a coleta e organização de dados de saúde; o diagnóstico de enfermagem inclui a identificação, concepção e verificação da resposta do cliente à saúde. Os resultados esperados referem-se a definições de metas personalizadas. A execução refere-se à implementação do plano de atendimento e obtenção dos resultados esperados; o processo evolutivo inclui comparar a resposta do cliente ao atendimento com os resultados esperados (Aprahamian et al., 2018).

A enfermagem assiste os pacientes com cuidados físicos, psicológicos e sociais, incluindo seu ambiente, seus cuidadores e familiares. À medida que a doença progride e os pacientes são completamente dependentes das necessidades básicas, a importância do cuidado torna-se mais importante. A importância da enfermagem no cuidado ao paciente com Doença de Alzheimer é ajudar a clientela a lidar com seu estado mental, até o atendimento clínico em hospitais especializados, para atender suas necessidades básicas de enfermagem (Leite et al., 2016).

A avaliação das capacidades funcionais do idoso pela equipe de enfermagem e equipes multiprofissionais tornou-se tão importante quanto o diagnóstico. A sistematização da assistência pode identificar problemas, auxiliar no planejamento, priorizar o suporte familiar, implantar e avaliar planos de cuidados individualizados e respeitar as diferentes fases da demência e o grau de dependência de cada idoso. Enfermagem além da avaliação funcional (Poltroniere et al., 2016).

O cuidado é essencial para os pacientes com DA, desde o diagnóstico até os estágios mais avançados. No início, o papel do enfermeiro era orientar a família nos cuidados adequados, visando minimizar o risco e manter sua integridade. No final da vida, além da orientação da equipe de enfermagem, os profissionais também devem estabelecer cuidados paliativos (Sales et al., 2016).

O profissional enfermeiro pode contribuir para tornar a ação em saúde resolutiva e duradoura, não apenas vista como um problema temporário, inclusive na atual estratégia de atenção à saúde, um plano que atenda às necessidades essenciais desta família (Santo et al., 2018).

A enfermagem tem a capacidade de trazer novas perspectivas sobre a doença aos pacientes, familiares e cuidadores, pois ela pode ser tratada mesmo que não tenha cura. A enfermagem pode melhorar a qualidade de vida, minimizar danos à saúde e evitar complicações. (Sayeg et al., 2019).

O profissional enfermeiro pode contribuir para tornar a ação em saúde resolutiva e duradoura, não apenas vista como um problema temporário, inclusive na atual estratégia de atenção à saúde, um plano que atenda às necessidades essenciais desta família (Santo et al., 2018).

A enfermagem tem a capacidade de trazer novas perspectivas sobre a doença aos pacientes, familiares e cuidadores, pois ela pode ser tratada mesmo que não tenha cura. A enfermagem pode melhorar a qualidade de vida, minimizar danos à saúde e evitar complicações. (Sayeg et al.,2019).

A assistência de enfermagem através de seus cuidados realizados é de grande e fundamental importância na vida do enfermo e da própria família, porém não é o suficiente, o profissional deve ter características como paciência, amor, comprometimento com o enfermo, possuir conhecimento e disponibilizar de uma educação continuada em prol de uma melhor qualidade nos cuidados prestados. (Costa et al., 2018).

O profissional de enfermagem tem como foco principal além da assistência adequada e necessária ao enfermo, disponibilizar uma melhor promoção referente à qualidade de vida do mesmo e da própria família, que acabam se tornando, em geral, os próprios cuidadores. Sendo igualmente responsabilidade do enfermeiro prover suporte e zelar pelos cuidadores. (Arruda et al., 2018). 
O tratamento ao indivíduo com DA só atingira melhores resultados quando essa doença possuir a devida importância, através da conscientização da população e quando compreendida a real necessidade da integração de cuidadores/família/equipe de enfermagem, podendo assim ser preparado um melhor plano de cuidados baseados no exame físico e clínico do paciente. (Leite et al., 2016).

\section{Considerações Finais}

Diante do que foi exposto, conclui-se então que os cuidados de enfermagem dispensados ao idoso acometido pela Doença de Alzheimer são essenciais para melhorar seu estado de saúde, promovendo, dessa forma, uma melhor qualidade de vida e autonomia, aumento sua sobrevida e fortalecimento do seu convívio familiar e social. Após a realização deste estudo, pode-se assim atentar e ampliar os conhecimentos sobre a importância da DA, permitindo um melhor entendimento sobre o assunto abordado, tendo em vista a grande relevância do papel dos profissionais de enfermagem através de seus conhecimentos e assistência prestados, já que a expectativa de vida de pessoas idosas vem aumentando com o passar dos anos e são os principais acometidos por essa enfermidade. Desta maneira, a elaboração deste trabalho possibilitou a compreensão da real importância em relação ao conhecimento científico sobre a DA, e a grande importância do profissional de enfermagem para que o idoso seja atendido dentro das condições ideais e o devido suporte e assistência à família que, na maioria dos casos, são os próprios responsáveis pelo ato de cuidar, proporcionando cuidados integrais e assim possibilitando uma redução de sofrimentos e esgotamentos, tendo como objetivo disponibilizar um estilo de vida mais equilibrado para ambos.

\section{Referências}

Alves, T. C. T. F. (2017). Pet do amiloide cerebral e da proteína tau no transtorno cognitivo leve.Rev. Psiquiatria Clinica, 43(2), $102-103$. Aprahamian, I. et al., (2018). Doença de Alzheimer: Revisão da Epidemiologia e Diagnóstico. Revista da Sociedade Brasileira de Clínica Médica,.6. Arruda et al., (2018). O familiar cuidador de portador de doença de Alzheimer participante de um grupo de ajuda mútua. Cienc. Cuid. Saude, 7(3), 339-345. Brunner, L. S et al., (2016). Cuidado de saúde do idoso. In: Brunner, L. S et al., Tratado de Enfermagem Médico-Cirúrgica. (12a ed.), LTD.

Bulla, L. C. Tsuruzono \& Eleni R. da S. (2016). Envelhecimento, Família e Políticas sociais. Revista Políticas Públicas. Universidade Católica do Rio Grande do Sul (PUC/RS). 14(1), 103-112.

Costa, E. G. Silva, M. C. C., Costa, M. L. G., \& Barros, A. L. S. (2017). Analise da deglutição em sujeitos portadores de doença de Alzheimer. Rev. Bras. Otorrinolaringol., 74(1).

Camacho, A. C. L. F et al., (2013). Revisão integrativa sobre os cuidados de enfermagem à pessoa com doença de Alzheimer e seus cuidadores. J. res.: fundam. care. 5(3), 186-193.

Dias, K. C. C. O., Lopes, M. E. L., Zaccara, A. A. L., Duarte, M. C. S., Morais, G. S. N. (2014). O cuidado de enfermagem direcionado para pessoa idosa: revisão integrativa. Rev enferm UFPE on line., 8(5), 1337-46.

Fechine, B. R. A. (2016). O processo de envelhecimento: as principais alterações que acontecem com o idoso com o passar dos anos. INTERSCIENCEPLACE, $1(20)$.

Gil, A. C. (2016). Como elaborar projeto de pesquisa.

Herrera-rivero, M. et al., (2016). in lymphocytes through cognitively normal ageing and neuropathology. An. Acad. Bras. Ciência, 85(4), 1489-1496.

Leite, C. D. S. M. et al. (2016). Conhecimento e intervenção do cuidador na doença de Alzheimer: uma revisão da literatura. J. bras. psiquiatr., 63(1), 48-56.

Pinto, A. V. et al., (2017). Tratamento farmacológico da doença de Alzheimer em idosos. Congresso Internacional de Envelhecimento Humano.

Poltroniere, S. et al., (2016). Doença de alzheimer e demandas de cuidados: o que os enfermeiros sabem? Rev. Gaúcha Enferm. 32(2), $270-278$.

Sales, A. C. S. et al. (2016) Conhecimento da equipe de enfermagem quanto aos cuidados com idoso portador da doença de Alzheimer. Revista de Enfermagem do CentroOeste Mineiro, 31(4).

Santo, F. H. E et al., (2018). Envelhecimento e morte na concepção dos idosos e profissionais de enfermagem: uma revisão integrativa. Revista Temática Kairós Gerontologia.

Sayeg, N. (2019). Como diagnosticar e tratar Doença de Alzheimer. R.B.M. 12(69), 12. 
Research, Society and Development, v. 11, n. 3, e36011326625, 2022

(CC BY 4.0) | ISSN 2525-3409 | DOI: http://dx.doi.org/10.33448/rsd-v11i3.26625

Silva, L. B. \& Souza, M. F. S. (2018). Os transtornos neuropsicológicos e cognitivos da doença de Alzheimer: A psicoterapia e a reabilitação neuropsicológica como tratamentos alternativos. Pretextos - Revista da Graduação em Psicologia da PUC Minas, 3(5).

Soares, J. S. \& Cândido, A. S. C. (2014). A assistência de enfermagem ao portador de Alzheimer e aos seus cuidadores: revisão integrativa do período 20052013. Revista Enfermagem Contemporânea. 3(1), 27-36.

Tiedemann, A. Lord, S. R. \& Sherrington, C. (2018). The Development and Validation of a Brief Performance-Based Fall Risk Assessment Tool for Use in Primary Care.The Journals of Gerontology Series A: Biological Sciences and Medical Sciences, 6(8), 896-903.

Oliveira C. A., \& Falcão M. E. O., (2014). Doença de Alzheimer: O enfermeiro frente às particularidades inerentes ao paciente e cuidador. Estação Científica - Juiz de Fora, no ${ }^{\circ} 12$, julho - dezembro. 